\title{
IDENTIFICATION OF GRAM POSITIVE BACTERIA INVOLVED IN YOLK SAC INFECTION
}

\author{
M.Rad, Z.Esmailnejad, Gh.Keleidari \\ Department of Pathobiology, School of veterinary medicine, Ferdowsi University of Mashhad, \\ P.O.Box: 1793 Mashhad 91775 Iran
}

\begin{abstract}
Yolk sac infection is one of the most important diseases in broiler chicken during first week of their life. The disease is prevalent in many flocks in Iran. As bacteria penetrate into yolk sac, immune system and antibodies can't destroy them, since yolk sac does not contain blood vessels. Therefore, identification of causal agents is important in prevention and control of the disease. In this report field outbreaks have been studied to determine the prevalent gram-positive bacterial types involved in yolk sac infection.

In this study, during one-year period from March 1999 till March 2000; 210 carcasses of broiler chicks with yolk sac infection from 21 broiler-breeding farms were put under investigation. These farms were in suburbs of Mashhad and Ghaen cities in Khorasan province in north east of Iran. Ten carcasses with yolk sac infection from each flock were sampled. The carcasses of the chicks were emaciated and smaller. The yolk sacs were found to be distended containing foul smelling yolk material. The color of the yolk varied from greenish yellow; dark brown to bright yellow. In some, the yolk material was almost completely solid whereas in some the consistency was watery.

Yolk sac material were collected aseptically from five carcasses and together cultured on different media for isolation of causative agents. The media used for first isolation were: blood agar with and without potassium tellorite, thioglicolate broth and manitol salt agar. Inoculated media incubated in aerobic and anaerobic conditions at $37^{\circ} \mathrm{C}$. Then developed colonies depended on macroscopic and microscopic appearance, subcultured on appropriate differential media for identification of genus and species of bacteria.
\end{abstract}

Results showed that streptococcus species were isolated from 21 flocks (100\% flocks). Staphylococcus species were recovered from 15 flocks (71.41\%). Bacillus cereus was isolated from 6 flocks (28.57\%), and Clostridium perfringens from 2 flocks (9.52\%). Pure culture of streptococcus spp. from 4 flocks (19/04\%) isolated. The prevalence of mixed infections were: staphylococcus and streptococcus spp. from 10 flocks (47/61\%); staphylococcus, streptococcus and clostridium spp. from one flock (4/76\%); staphylococcus, streptococcus, bacillus and clostridium spp. from one flock (4/76\%); staphylococcus, streptococcus and bacillus spp. from 3 flocks (14/28\%); streptococcus and bacillus spp. from 2 flocks (9/52\%). Isolated streptococcus species included: avium, durans, faecium, faecalis, porcinus and isolated staphylococcus species included: aureus, hycus, gallinarus and epidermidis.

However, isolated bacteria in this study contain enzymatic systems of lipase, protease and lecithinase, which are able to digest protein of yolk, so they provide opportunity for other invader bacteria to complicate infection. The present investigation throws light on the possibility of severity of yolk sac infection and chick mortality in poultry industry due to bacterial flora, if proper sanitary and strict precautionary measures were not undertaken at every step, before, during and soon after hatching of eggs. 